\title{
EXISTENCE OF A SIMPLE AND EQUITABLE FAIR DIVISION: A SHORT PROOF
}

\author{
GUILLAUME CHÈZE
}

\begin{abstract}
In this note we study how to share a good between $n$ players in a simple and equitable way. We give a short proof for the existence of such fair divisions.
\end{abstract}

In this note we study a fair division problem. Fair divisions problems are sometimes called cake cutting problems. These kinds of problems appear when we study division of land, time or computer memory between different agents with different point of view. This problem is old: the "cut and choose" algorithm already appears in the Bible. In a more scientific way it has been formulated by Steinhaus in 1948, see [Ste48. Nowadays, there exists several articles, see e.g. DS61, BT95, RW97, BJK13, and books, see e.g. [RW98, BT96, Pro16, Bar05, about this topic. These results appears in the mathematics, economics, political science, artificial intelligence and computer science literature.

In this note, our heterogeneous good, e.g. a cake, will be represented by the interval $[0 ; 1]$. We consider $n$ players and we associate to each player a non-atomic probability measure $\mu_{i}$ on the interval $X=[0 ; 1]$ with density $f_{i}$. These measures represent the utility functions of the player. The set $X$ represents the cake and we want to get a partition of $X=X_{1} \sqcup \ldots \sqcup X_{n}$, where the $i$-th player get $X_{i}$.

In this situation several notions of fair division exists:

- Proportional division: $\forall i, \mu_{i}\left(X_{i}\right) \geq 1 / n$.

- Exact division: $\forall i, \forall j, \mu_{i}\left(X_{j}\right)=1 / n$.

- Envy-free division: $\forall i, \forall j, \mu_{i}\left(X_{i}\right) \geq \mu_{i}\left(X_{j}\right)$.

- Equitable division: $\forall i, \forall j, \mu_{i}\left(X_{i}\right)=\mu_{j}\left(X_{j}\right)$.

All these fair divisions are possible, see e.g [Ste48, DS61, BT96, RW98, CDP13. The minimal number of cuts in order to get a fair division has also been studied. For a proportional fair division $n-1$ cuts are sufficient. This means that for all $i$ there exists an index $j$ such that $X_{i}=\left[x_{j}, x_{j+1}\right]$, where $x_{0}=0, x_{n}=1$. A fair division where each $X_{i}$ is an interval is called a simple fair division. The BanachKnaster algorithm given in Ste48] shows how to get a simple proportional fair division.

Stromquist and Woodall have shown that we can always get a simple envy-free fair division, see [Str80, Str81, Woo80. These two different proofs use the Brouwer

Key words and phrases. Fair divisions; Cake cutting; Equitable divisions; Borsuk-Ulam theorem. 
fixed point theorem.

For an exact division $n(n-1)$ cuts are sufficient and this bound is also optimal, see Alo87. This means that we cannot always get an exact division where each $X_{i}$ is an interval. The strategy used by Alon to prove his result relies on a general version of the Borsuk-Ulam theorem.

The Borsuk-Ulam theorem is a classical tool in fair division. Indeed, it implies the Ham sandwich theorem see DS61, and it is also used to get a consensus halving, see e.g. DS61, SS03. Consensus halving means that we want to get two sets $A$ and $B$ such that $[0,1]=A \sqcup B$ and for all $i, \mu_{i}(A)=\mu_{i}(B)$. This result corresponds to the Hobby-Rice theorem and a proof using the Borsuk-Ulam theorem has also been given by Pinkus, see Pin76. As Borsuk-Ulam theorem implies the Brouwer fixed point theorem we can say that the existence of a simple and envy-free fair division relies on the Borsuk-Ulam theorem. This suggests that the Borsuk-Ulam theorem is an ubiquitous tool in cake-cutting problems.

However existing proofs about equitable and simple fair division do not use this theorem. Proofs have been given by Cechlárová, Doboš and Pillárová in [CDP13. and independently by Aumann and Dombb in AD15. In [CDP13 the authors have proved that for all permutations $\sigma \in \mathcal{S}_{n}$ the system of equations:

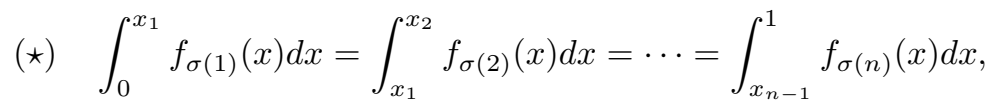

has a solution with $0 \leq x_{1} \leq x_{2} \leq \cdots \leq x_{n-1} \leq 1$. The permutation $\sigma$ corresponds to the players' order: the $\sigma(i)$-th player get the interval $\left[x_{i-1}, x_{i}\right]$.

The proof given in CDP13 is based on the notion of the generalized inverse of a function. In AD15 the authors give another proof of this result which uses a compactness argument.

Thus we have two different proofs but these proofs do not use the classical tool: the Borsuk-Ulam theorem.

In the following we show how to get the existence of an equitable and simple fair division shortly thanks to this classical theorem.

Theorem 1. For all densities function $f_{i}$ and all permutations $\sigma \in \mathcal{S}_{n}$ the system of equations $(\star)$ has a solution. This means that there exists an equitable and simple fair division.

Our proof relies on the Borsuk-Ulam theorem: If $f: S^{k} \rightarrow \mathbb{R}^{k}$ is continuous and antipodal $(f(-x)=-f(x))$ then there exists $x_{0} \in S^{k}$ such that $f\left(x_{0}\right)=0$.

Proof. We consider the sphere $S^{n-1}=\left\{e=\left(e_{1}, \ldots, e_{n}\right) \in \mathbb{R}^{n} \mid \sum_{i=1}^{n} e_{i}^{2}=1\right\}$ and the function

$$
\begin{aligned}
f: S^{n-1} & \rightarrow \mathbb{R}^{n-1} \\
e & \longmapsto\left(F_{1}(e), \ldots, F_{n-1}(e)\right)
\end{aligned}
$$

where

$$
F_{i}(e)=\operatorname{sgn}\left(e_{i+1}\right) \cdot \int_{e_{1}^{2}+\cdots+e_{i}^{2}}^{e_{1}^{2}+\cdots+e_{i}^{2}+e_{i+1}^{2}} f_{\sigma(i+1)}(x) d x-\operatorname{sgn}\left(e_{1}\right) \cdot \int_{0}^{e_{1}^{2}} f_{\sigma(1)}(x) d x .
$$

The function $f$ is continuous and antipodal, thus by the Borsuk-Ulam theorem there exists $\tilde{e} \in S$ such that $f(\tilde{e})=0$. 
We set $x_{0}=0$ and $x_{i}=x_{i-1}+\tilde{e}_{i}^{2}$ and we get the desired solution for the system $(\star)$.

\section{REFERENCES}

[AD15] Yonatan Aumann and Yair Dombb. The efficiency of fair division with connected pieces. ACM Trans. Econ. Comput., 3(4):Art. 23, 16, 2015.

[Alo87] Noga Alon. Splitting necklaces. Adv. in Math., 63(3):247-253, 1987.

[Bar05] Julius B. Barbanel. The geometry of efficient fair division. Cambridge University Press, 2005.

[BJK13] Steven J. Brams, Michael A. Jones, and Christian Klamler. $N$-person cake-cutting: There may be no perfect division. The American Mathematical Monthly, 120(1):35-47, 2013.

[BT95] Steven J. Brams and Alan D. Taylor. An envy-free cake division protocol. The American Mathematical Monthly, 102(1):9-18, 1995.

[BT96] Steven J. Brams and Alan D. Taylor. Fair division - from cake-cutting to dispute resolution. Cambridge University Press, 1996.

[CDP13] Katarína Cechlárová, Jozef Doboš, and Eva Pillárová. On the existence of equitable cake divisions. Inform. Sci., 228:239-245, 2013.

[DS61] L.E. Dubins and E. H. Spanier. How to cut a cake fairly. The American Mathematical Monthly, 68(1):1-17, 1961.

[Pin76] Allan Pinkus. A simple proof of the Hobby-Rice theorem. Proc. Amer. Math. Soc., 60:82-84 (1977), 1976.

[Pro16] Ariel D. Procaccia. Cake cutting algorithms. In F. Brandt, V. Conitzer, U. Endriss, J. Lang, and A. D. Procaccia, editors, Handbook of Computational Social Choice, chapter 13. Cambridge University Press, 2016.

[RW97] Jack M. Robertson and William A. Webb. Near exact and envy-free cake division. Ars Combinatoria, 45:97-108, 1997.

[RW98] Jack M. Robertson and William A. Webb. Cake-cutting algorithms - be fair if you can. A K Peters, 1998.

[SS03] Forest W. Simmons and Francis Edward Su. Consensus-halving via theorems of BorsukUlam and Tucker. Math. Social Sci., 45(1):15-25, 2003.

[Ste48] Hugo Steinhaus. The problem of fair division. Econometrica, 16(1):101-104, January 1948.

[Str80] Walter Stromquist. How to cut a cake fairly. Amer. Math. Monthly, 87(8):640-644, 1980.

[Str81] Walter Stromquist. Addendum to: "How to cut a cake fairly" [Amer. Math. Monthly 87 (1980), no. 8, 640-644; MR 81m:05016]. Amer. Math. Monthly, 88(8):613-614, 1981.

[Woo80] D.R Woodall. Dividing a cake fairly. Journal of Mathematical Analysis and Applications, $78(1): 233-247,1980$.

Guillaume Chèze: Institut de Mathématiques de Toulouse, Université Paul Sabatier Toulouse 3, MIP BÂT. 1R3, 31062 TOUlousE Cedex 9, France

E-mail address: guillaume.cheze@math.univ-toulouse.fr 